\title{
Influencias del muralismo mexicano en los muros de Costa Rica
}

Mauricio César Ramírez Sánchez Académico

Univesidad Nacional Autónoma

México

\section{Resumen}

El siglo Xx inició con una serie de cambios, tanto en el terreno tecnológico como en el político, que se generalizaron a nivel mundial. En el terreno del arte también surgieron inquietudes de renovación. Como prueba de ello se cuentan las manifestaciones artísticas conocidas como vanguardias, que se extendieron por todo el mundo; aunque en algunos países adquirieron características propias, e incluso originaron movimientos únicos. Ejemplo de ello fue el muralismo mexicano, en la década de los vein-

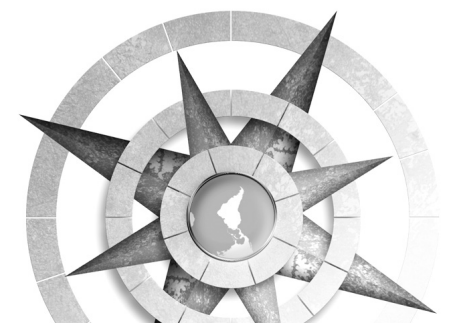

tes, que impactó en distintos momentos en algunos países de América. Costa Rica no escapó a este influjo.

Palabras clave: muralismo mexicano, vanguardias, muralistas costarricenses, muralistas mexicanos, Cátedra del exilio, Temas de Nuestra América

\section{Abstract}

The $20^{\text {th }}$. century began with a chain of changes in the technological as well as the political fields that became generalized worldwide. Concerns of renewal also emerged in Arts. The artistic manifestations known as avant-gardes evidenced it. Avant-gardes spread out all over the world; and in some countries they achieved their own characteristics and even created unique movements. As an exemplar, Mexican muralism, in the 1920's, impacted at different times in some American countries. Costa Rica did not escape its influence. 
Keywords: Mexican muralism; avantgardes, Costa Rican muralism, Mexican muralism, Exile Reseach Group, Temas de Nuestra América

El siglo xx inició con una serie de cambios, tanto en el terreno tecnológico como en el político, que se generalizaron a nivel mundial. En el terreno del arte también se hicieron presentes las inquietudes de renovación; prueba de ello son las manifestaciones artísticas conocidas como vanguardias, que se extendieron por todo el mundo; aunque en algunos países adquirieron características propias, e incluso originaron movimientos únicos. Lo cierto fue que sumándose a ellas u oponiéndoseles, éstas terminaron por hacerse presentes en la vida cotidiana.

A pesar de la lejanía que en la actualidad puede considerarse que tienen las inquietudes artísticas que se vivieron durante la primera mitad del siglo $\mathrm{xx}$ y que ahora suelen englobarse simplemente como vanguardias, cabe decir que una particularidad de éstas fue la movilidad que se dio tanto de ideas como de personas, con lo que terminaron por tener un impacto universal.

Aunque la mayoría de estas vanguardias tuvo su origen en territorio europeo, es posible afirmar que en el continente americano hubo manifestaciones artísticas que terminaron por ser un foco de atracción para los artistas del continente. ${ }^{1}$ Ejemplo de ello fue el muralismo que surgió en México, en la década de los veintes; pues, impactó en distintos momentos en algunos países de América. Ello ha llevado a Marta Traba a señalar que "las relecturas críticas más acertadas y desapasionadas que actualmente se hacen del muralismo, o Escuela Mexicana, coinciden en considerarlo como el movimiento más importante de la plástica continental de comienzos de siglo". 2 Al mismo tiempo, es factible señalar que éste ofreció la oportunidad de romper con la influencia europea que había permeado en el arte, en lo que resultaron fundamentales los conflictos europeos, pues llevaron a algunos artistas a evitar viajar a ese continente.

El impacto de los muralistas mexicanos, -por lo menos de los más representativos-, José Clemente Orozco,

1 Algunas de las manifestaciones que se vivieron en el arte del continente americano pueden verse en: Ana María de Moraes Belluzzo, et.al., Modernidade: vanguardias artísticas na America Latina, Sao Paulo, Brasil, Universidad Estadual Paulista Julio de Mesquita Filho, 1990; Daniel Noemi Voinmaa, Revoluciones que no fueron: ¿arte o política? Más allá de realismo y vanguardias en América Latina, Ecuador y Chile: 1924-1938, España, Cuarto Propio, 2013; y Esther Acevedo, México y la invención del arte latinoamericano 1910-1950, México, SRE, 2011.

2 Marta Traba, Arte de América Latina 1900-1980, NUEVA York, Banco Interamericano de Desarrollo, 1994, p.14. 
Diego Rivera y David Alfaro Siqueiros se debió a las diversas obras que realizaron fuera del país; sobre todo destaca la incursión de Siqueiros por diferentes países de Latinoamérica, en los que dictaba conferencias, y llegó a desarrollar obras que hoy resultan emblemáticas dentro de la historia del arte. ${ }^{3}$

En lo que se refiere a Costa Rica algunos de sus artistas no escaparon a la influencia del muralismo mexicano. ${ }^{4}$ Aunque, la atracción de esta corriente se manifestó hasta los años cuarenta; antes no existen referencias que indiquen que los artistas se interesaban por la realización de obras de gran formato.

Corresponderá al artista Francisco Amighetti la introducción del muralismo al arte costarricense. De hecho, puede afirmarse que fue un artista interesado en la renovación constante del arte

3 En Argentina realizó Ejercicio plástico, en 1933; en Chile pintó Muerte al invasor, en 1941; en Cuba Alegoría de la igualdad racial en Cuba y El nuevo día de las democracias, en 1943; en Estados Unidos, en 1932, elaboró Un mitin obrero, La América tropical oprimida y destrozada por los imperialismos y Retrato actual de México.

4 Un caso interesante será el de Francisco Zúñiga Chavarría quien en 1936 viajó a México, donde permaneció el resto de su vida y desarrolló la mayoría de sus obras; entre ellas, diferentes murales. Véase Ariel Zúñiga, Francisco Zuninga: desde el umbral: from the threshold, México, Fundación Zúñiga Laborde, 2015; y también de Zúñiga, Francisco Zúniga: homenaje nacional, México, Conaculta, 1994. costarricense, por lo que Manuel de la Cruz González Luján llegó a expresar: "en la revisión de mis recuerdos, de un acertado caricaturista Amighetti salta a la pintura, corren los últimos años de la década de los veinte. Más y mejor informado que otros sucumbe ante la forma momparnasiana introduciendo el cubismo picassiano en Costa Rica. Produce entonces una serie de dibujos y pequeños cuadritos en los que se manifiesta la vanguardia renovadora." ${ }^{5}$ Esa misma necesidad de renovación lo llevó a incursionar por diferentes técnicas artísticas. Francisco Amighetti no limitó su interés en el estudio de los diversos caminos que el arte estaba siguiendo en la primera mitad del siglo xx; pues su necesidad de enriquecer su visión pictórica lo hizo recorrer diferentes países del continente americano. Parte de sus vivencias que experimentara en estos

5 M. de la Cruz González Luján, Francisco Amighetti el pintor, Exposición Retrospectiva y Contextual, Museo de Arte Costarricense, marzo de 1967. Cfr. Costa Rica en el arte. Colección de artes plásticas. Banco Central de Costa Rica, San José de Costa Rica, Museo Banco Central de Costa Rica, 1990, en que se dice: "Entre los artistas que desde 1930 mantuvieron su independencia y un estilo personal, se halla la indiscutible figura de Francisco Amighetti. Si bien este artista trabajó una temática también regionalista, se distinguió del resto por tener la capacidad de ir más allá del simple hecho de reproducir miméticamente el tema de la casa de adobes. Hacia 1940 emprendió una obra encaminada hacia la experimentación del mural al fresco, sin abandonar el óleo y otras técnicas. A partir de 1968, última etapa de su producción, incursionó en la cromoxilografía”, p.123. 
países le inspiraron a escribir su obra Francisco y los caminos en donde a manera de presentación advertía:

Este libro se titula Francisco y los caminos, porque los abarca todos, tenía que situar mis andanzas, y así lo hice. Escribo cosas sin importancia. Algunas de las gentes que encontré en mis viajes, tenían una vida con más color y claroscuro, con más drama que mi pálida existencia. Esos hombres y mujeres dejaron en mi libro muchas de sus palabras agitadas, su llanto, su prolongado silencio, su algarabía, y la plástica de su perfil en la sombra. ${ }^{6}$

De esta manera, en su libro hace referencia a algunas de sus vivencias durante su paso por Panamá, Buenos Aires, Estados Unidos, El Salvador, Perú y Guatemala.

Llama la atención que entre dichos recuerdos sólo indirectamente haga referencia a su paso por México, ${ }^{7}$ siendo que

6 Francisco Amighetti, Francisco y los caminos, San José de Costa Rica, Editorial Costa Rica, 1980, p. 9.

7 A México sólo se refirió al recordar un cuadro que realizó a su casera en este país; y menciona a Diego Rivera al ponerlo en un comentario del artista Dorothy Brett: "deje la literatura -dijo-. Eso de usar tinta y pluma es sucio y feo; en cambio en el oficio de pintar todo es atrayente: telas tensas y blancas, pinceles del pelo de animales exóticos, espátulas relucientes, paletas de nogal, aluminio y el vinculo que Amighetti tendrá con México no puede considerarse menor; ya que, en 1947 emprendió un viaje a este país para ver de forma directa los murales que se habían realizado en él; pero, sobre todo, para estudiar la técnica mural. El lugar en que llevó a cabo sus estudios fue la escuela La Esmeralda, bajo la tutela del muralista Federico Cantú. ${ }^{8}$

A ello debe agregarse que en México dejó ejemplo de su trabajo como grabador, ${ }^{9}$ lo que resulta significativo, pues también en este país contaba con una larga tradición en el grabado, incluso durante su paso por México se encontraba en su apogeo el Taller de Gráfica Popular, que congrega a los artistas más representativos del país. ${ }^{10}$

porcelana, papeles de todas las texturas y todos los tonos, materiales e instrumentos todos preciosos, sólo falta ser artista, pero no hay que sufrir por eso. Considérese usted tan bueno como Picasso o Diego Rivera y estará tranquilo".

8 Federico Cantú había ingresado como profesor en La Esmeralda, desde 1943, en cuanto a su actividad artística además del muralismo, practicó la pintura de caballete, el grabado, la escultura, pero sobre todo destacó en el dibujo. Sobre él pueden verse: Federico Cantú, Federico Cantú, Monterrey, Nuevo León, Universidad Autónoma de Nuevo León, 2008; y también de Federico Cantú, Seis décadas, México, UNAM, 1984.

9 De su autoría publicó el libro, Francisco en Harlem, México, Galería de Arte Centroamericano, 1947. De igual manera realizó ilustraciones para el libro de Ricardo Posas Arciniega, Zís ma ise: (Yo voy a decir), México, Junta de Protección de las Razas Aborígenes de la Nación, 1947.

10 El Taller de Gráfica Popular se crea en 1937, bajo la iniciativa de Leopoldo Méndez, Pablo 
De regreso a Costa Rica, se incorporó como profesor de la Escuela de Bellas Artes, en la que impartió un curso sobre muralismo, en 1947, con lo que puede establecerse que lo visto y aprendido en México habían dejado una huella en él:

[...] de los muralistas mexicanos, Orozco el filósofo, Siqueiros el revolucionario y Rivera, el folklorista histórico, Amighetti experimenta la presión de este último en mayor grado. Tamayo en cambio, el eslabón entre el presente y el pasado, no ejerce ninguna influencia en él. Esto de las influencias es no sólo natural sino necesario. Picasso decía que en nuestra época es difícil encontrar a alguien que no sufra influencias de alguien ya que éstas o el aprovechamiento de los descubrimientos es lo que constituye en suma el progreso, que lo que importa no es influenciarse, sino influenciarse bien. ${ }^{11}$

O’Higgins y Luis Arenal. En dicha organización se congregaron númerosos artistas tanto nacionales como extranjeros, que se preocuparon por contribuir con su obra en los reclamos sociales, para lo que se valieron de las diferentes técnicas del grabado. Algunos de los trabajos en que puede verse la riqueza artística que surgió de dicha organización son: Helga Prignitz-Poda, Taller de Gráfica Popular, Berlín, Ibero-Amerikanisches Institut-Preussischer Kulturbesitz, 2002. y Humberto Musacchio, El Taller de Gráfica Popular, México, FCE, 2007.

11 Alberto Calderón Vega, "El salvamento del Mural de la Segunda República: recuperar el patrimonio y la
Resulta claro que para él, el muralismo podía tener una trascendencia en su país, pues el mismo año sus preocupaciones teóricas se vieron reflejadas en la práctica al realizar para la familia González Feo la reproducción de los murales que el Giotto pintó en la Capilla de los Scrovegni, en Padua. ${ }^{12}$ Pero estas primeras obras sólo pueden verse como una etapa de experimentación, que, sin embargo, comenzaría a atraer la atención de otros artistas, entre los que destacan Margarita Bartheau, quien colaboró con él en la mayoría de sus proyectos. Sobre la artista, el propio Amighetti llegó a decir: "ayer estuve ordenando los colores que uso en la pintura al fresco y me llegó su recuerdo. Ella con la pasión que la caracterizaba se lanzó a aprender aquella técnica que viene más allá del Renacimiento y que la épica del mural mexicano revivió". ${ }^{33}$ Participó con él

memoria histórica de un pueblo," en Temas de Nuestra América, Costa Rica, enero-junio de 2010, p. 29.

12 Carlos Guillermo Montero, Amighetti: 60 años de labor artística, San José de Costa Rica, Museo de Arte Costarricense, 1988, p. 62. Afirma que "antes de partir hacia México Amighetti había llevado a cabo los primeros ensayos," haciendo referencia precisamente a las copias que realizó para la familia González Feo.

13 Francisco Amighetti, Francisco en Costa Rica, San José de Costa Rica, Editorial Costa Rica, 1980, p. 192. Cfr. Montero, op. cit., señala que a su vez Margarita Bertheau, "quien compartió la experiencia del muralismo con Amighetti, para testimoniarlo, pintó su retrato en uno de los murales del Colegio Saint Francis, que lleva el nombre del pintor y del santo; este detalle 
en el arranque del muralismo en Costa Rica, aunque esas primeras pruebas no estuvieron exentas de errores. ${ }^{14}$

\section{La importancia del muralismo cobraría} interés a partir de los propios sucesos sociales que empiezan a manifestarse desde 1947 y que tendrán su detonante con la Guerra Civil de 1948. De esta manera, el muralismo que comenzaba a surgir en Costa Rica estableció una liga con el arte mexicano, pues este último nació como resultado del conflicto armado que había enfrentado en la segunda década del siglo. ${ }^{15}$

recuerda el Autorretrato con paleta de la colección Yankelewitz", p. 70.

14 Amighetti, Francisco en Costa.., op.cit., al recordar a Margarita Bartheau, quien para ese momento ya había muerto, señala: "habitaba yo entonces en San José donde está la sucursal del Banco Nacional, una casa vieja pero con paredes ideales para empezar el fresco en Costa Rica. Allí con su trazo libre empezamos a pintar, pero algunos colores que no eran propios para el fresco, los destruyeron las calorías latentes de la cal".

15 El movimiento armado de 1910 modificará el aspecto cultural del país; pero, al mismo tiempo terminó por dar una imagen hacia el exterior; por ello Traba, op. cit., dice: "El muralismo mexicano, directa o indirectamente, contribuyó a que los artistas proclives a explotar la temática social, indígena o mestiza, negra o mulata, quedaran sólidamente inscritos en los marcos históricos comprendidos entre 1920 y 1940. Paralelamente, otro grupo de artistas intentó, en el mismo periodo, crear un espacio cultural donde se emprendiera, antes que cualquier otra cosa, la transformación radical de la imagen académica y decimonónica. En ellos, el lenguaje plástico ocupó un lugar importante. Se define el camino de la modernización de la imagen, y el continente se reincorpora
El mural La agricultura es significativo por ser el primer mural que se realizó al fresco en Costa Rica, bajo la influencia del muralismo mexicano. al sistema de reinvenciones formales que caracteriza a la plástica del siglo XX", p. 50. 


\section{Figura 1. \\ La agricultura}

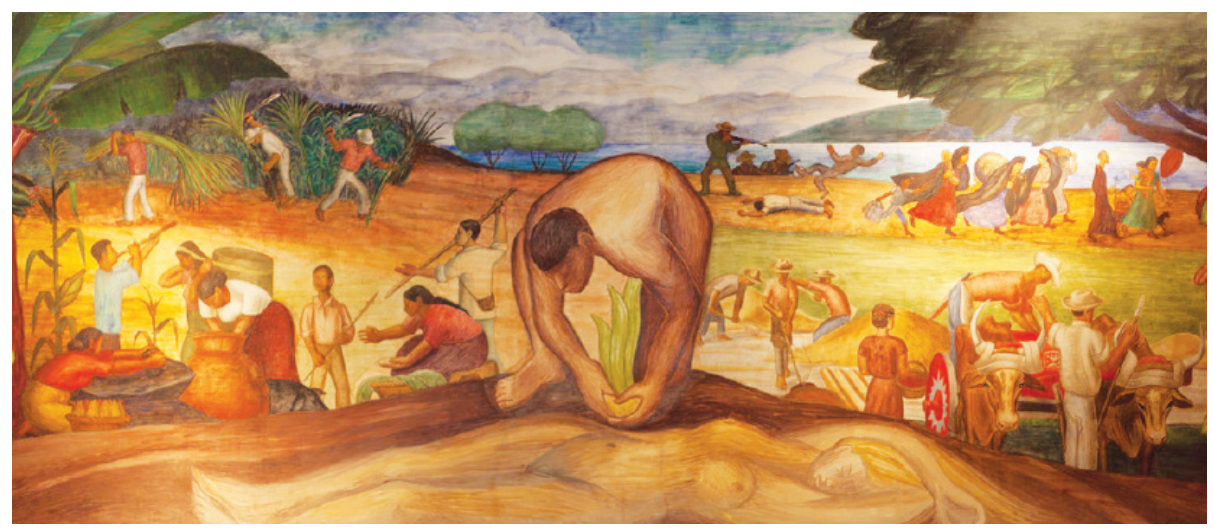

Autor: Francisco Amighetti, 1948. Imagen tomada de: http://upload.wikimedia.org/wikipedia/commons/thumb/e/e4/CRart.LaAgric.Amighett.jpg/700px-CRart.LaAgric.Amighett.jpg

Cabe señalar que en esta obra es evidente su paso por suelo mexicano y, sobre todo, establece una liga directa con la obra que Diego Rivera creó entre 1926 y 1927, en la exhacienda de Chapingo, ${ }^{16}$ la cual para ese entonces ya funcionaba como escuela, y que lleva por nombre general Canto a la tierra y a los que trabajan y liberan o Enseñar la explotación de la tierra, no la del hombre y Evolución de la tierra y evolución de los hombres.

16 Sobre los murales que Diego Rivera realizó en la hacienda de Chapingo se ha escrito mucho; pues, son considerados como obras emblemáticas del muralismo mexicano. No obstante, una obra que sigue siendo referencia obligada es la de Antonio Rodríguez, Canto a la tierra. Los murales de Diego Rivera en la Capilla de Chapingo, México, Universidad Autónoma de Chapingo, 1986, [ilus.].
Diego Rivera se encontraba realizando la decoración de la Secretaría de Educación Pública, trabajo que suspendió por viajar a Rusia y por los murales de Chapingo. Como parte de este proyecto, decoró la rectoría y la excapilla de la hacienda. En la primera, resaltan las alusiones al mal y al buen gobierno; alude a los despojos de que habían sido víctimas los campesinos. Mientras que en el segundo, el reparto de la tierra aparece como uno de los logros de la Revolución.

Lo representativo de las obras que crea en este lugar, y que seguramente fueron vistas por Francisco Amighetti durante su estancia en el país, se encuentra en los murales que decoran la excapilla. 
En éstos, Diego Rivera contrapone la evolución natural a los acontecimientos armados vividos por México y que habían llevado a la conquista de la tierra. En ellas destacan las figuras de Emiliano Zapata y de Otilio Montaño, como mártires de la lucha por la tierra. Parte importante del ideal de la escuela está representado en la sección denominada "la tierra fecunda", que se encuentra en el ábside de la capilla. En éste se ve a la tierra representada por una mujer, -retrato de Lupe Marín-, de cuya mano derecha brota una planta. Ésta se complementa con la presencia del viento, el agua y el fuego. Aparece la tecnología, pero no está destinada a la explotación del hombre, sino a estar bajo su dominio y facilitarle la vida.

Por su parte Francisco Amighetti, en La agricultura, coloca al centro a una mujer desnuda recostada, que representa a la tierra. Sobre ella se encuentra un hombre, también desnudo, depositando una semilla en la tierra; y de la unión de ambos, surge la vida, simbolizada por la germinación de la semilla. ${ }^{17}$

Detrás de estas figuras, el artista destaca los principales productos agrícolas que exportaba Costa Rica, por lo que eran

17 Simbólicamente, la germinación de una semilla puede verse como la multiplicación de la vida y el nacimiento de una nueva época. Véase Jean Chevalier y Alain Gheerbrant, Diccionario de los simbolos, Barcelona, Herder, 1999, p. 538. fundamentales para su economía. Del lado derecho se incluye el maíz, desde que es planta, hasta que se convierte en alimento. En esta misma sección se resalta la importancia que tienen las plantaciones de plátanos. En el fondo de la imagen se observa a tres hombres en la zafra de la caña, que era utilizada para la producción de azúcar y, por lo tanto, de gran valor para el país.

En el lado izquierdo se alude al principal producto agrícola de Costa Rica: el café. Así, se ven los montones de granos que se han puesto a secar para, posteriormente, ser encostalados y transportarlos en una carreta tirada por bueyes. La producción del café, para la economía del país, era tan importante que entre las primeras acciones del gobierno estuvo la de tratar de controlarla a través de la transformación del Instituto de Defensa del Café en Oficina del Café, la cual "regularía los precios que debían pagarse a los productores e intervendría en todo lo que tuviera que ver con las relaciones entre productores, beneficiados y exportadores ${ }^{18}$.

En el fondo de la imagen se alcanza a ver a un grupo de mujeres que huyen con sus hijos y pertenecías a cuestas. Éstas son acompañadas por un fraile que sostiene

18 Héctor Pérez Brignoli, Breve historia contemporánea de Costa Rica, México, FCE, 2002, p.132. 
en su mano derecha un crucifijo. Detrás de ellas, un grupo de hombres armados dispara sobre unos indefensos; uno de ellos se encuentra tendido sobre el piso, por lo que seguramente se ha concretado su asesinato. Esta escena ha sido explicada como "campesinos heridos por los mulatos que se robaban el cacao en la época colonial". ${ }^{19}$ El mural La agricultura fue realizado en la Casa Presidencial, al poco tiempo de haberse concluido la Guerra Civil de $1948,{ }^{20}$ por lo que no resultaría extraño que el artista introdujera una referencia a ese hecho.

La figura del religioso en la obra también puede explicarse si se recuerda que durante la Guerra Civil fue de gran importancia el sacerdote Benjamín Núñez Vargas, quien se desarrolló como Capellán del Ejército de Liberación Nacional; pero, sobre todo, fue de gran provecho en las negociaciones que llevaron a la pacificación del país

19 Peggy Taylor Filloy, "Traslado de los murales al fresco de la antigua Casa Presidencial al Museo de Arte Costarricense," en Revista Pensamiento Actual, San José de Costa Rica, vol. 5, núm. 6, 2005, p. 100.

20 Sobre la ejecución de este mural, véase Carlos Guillermo Montero, Amighetti: 60 años de labor artística, San José de Costa Rica, Museo de Arte Costarricense, 1988, quien señala: "al volver al país encuentra su oportunidad en la Casa Presidencial: José Figueres les ofrece materiales y comida, ésta que comparten con los policías mientras llevan a cabo el mural titulado La Agricultura. Amighetti enseñó la técnica a Margarita Bertheau y casi siempre trabajaron juntos", p.62. y a la conformación de la Segunda República. ${ }^{21}$

Resulta claro que al darse cualquier confrontación armada, lo primero que se ve afectada es la producción económica de un país. De esta manera, la obra de Amighetti puede entenderse como un momento de reconstrucción en que acababa de entrar el país, con lo que se verían beneficiados todos los costarricenses, por lo menos ideológicamente, para tratar de consolidar la unificación de Costa Rica.

En la Casa Presidencial, Luis Daell elaboró el mismo año un mural al que llamó La piedad.

$21 \mathrm{Al}$ respecto, puede verse el texto de Rodrigo Carreras, "Reseña Biográfica del Rev. Dr. Benjamín Núñez Vargas: Sacerdote, Sindicalista, Capellán, Diplomático, Académico y Soñador,” en El espíritu del 48. Dedicado a preservar los valores e ideales de la Revolución de 1948. http://elespiritudel48.org/ resena-biografica-del-rev-dr-benjamin-nunez-vargas/ (Consultado: 25 de agosto de 2016). En dicho texto, no sólo se alude al papel desempeñado por el sacerdote durante el conflicto armado, sino a su larga trayectoria política dentro y fuera de Costa Rica. 


\section{Figura 2.}

La piedad

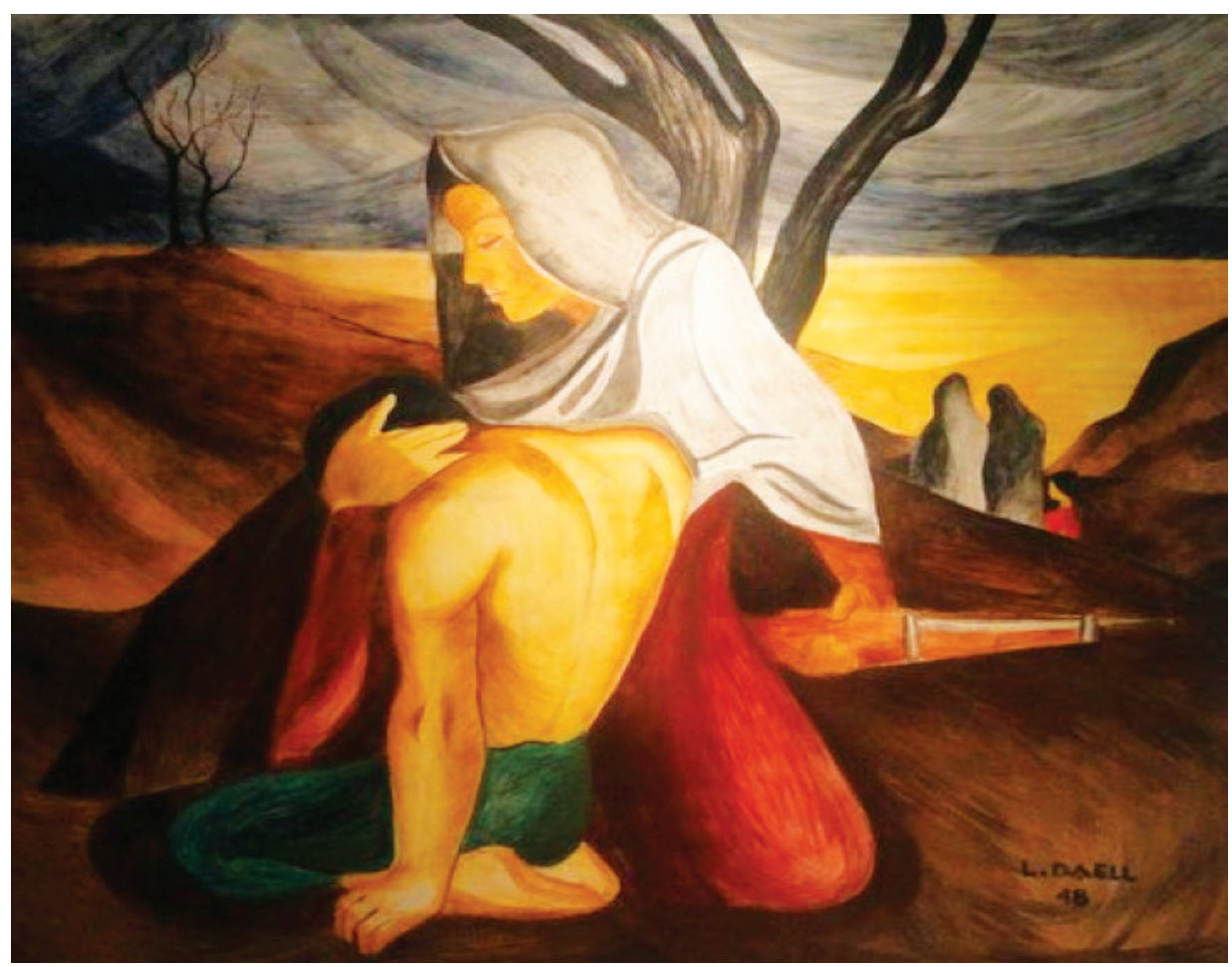

Autor: Luis Daell, 1948. Imagen tomada de: https://media-cdn.tripadvisor.com/media/photo-s/04/09/ad/77/museo-de-arte-costarricense.jpg
Cabe señalar, que Daell había estudiado la técnica del fresco directamente con Francisco Amighetti, ${ }^{22}$ durante el curso impartido en 1947 en Bellas Artes. Con

22 Destacable resulta que sea en el propio año de 1947 que Daell haga los estudios de la técnica al fresco con Francisco Amighetti; pues, como se vio durante ese mismo año, la había estudiado en México. Llama la atención que después de la realización de esta obra, Daell se sintiera atraído por profundizar en el muralismo, por lo que viajó a México y Estados Unidos. Véase, Taylor, op. cit., p. 99. 
ello, puede decirse que el interés por el muralismo estaba popularizándose entre los artistas, pero también se buscaba ir perfeccionando la técnica. ${ }^{23}$

Si en la obra de Amighetti puede notarse una relación velada con la Guerra Civil del 48, en la obra de Luis Daell la relación es clara. En lo que se refiere a la imagen tenemos la representación de un paisaje desolado, como producto de la guerra, en el que las mujeres quedan a la espera de los hijos y esposos que han partido para combatir. En primer plano se ve a una mujer arrodillada, que sujeta con el brazo derecho el cuerpo desfallecido de un hombre, que lleva el torso desnudo. Con ello se alude a los desastres del conflicto por los que el país acababa de pasar; pues, al mismo tiempo la mujer sostiene con la mano izquierda un arma, como símbolo de los enfrentamientos que recientemente habían sucedido entre los propios costarricenses.

23 Dentro de su etapa de experimentación, hizo la obra titulada El mercado, en la Facultad de Bellas Artes. Es posible que dicha obra fuera resultado de poner en práctica lo aprendido en el curso que había impartido Francisco Amighetti, lo que explicaría, al mismo tiempo su desaparición. No obstante, a través de una fotografía que se conserva en la que Luis Daell se encuentra posando frente a este mural se alcanza a ver que utilizó una serie de figuras robustas, para representar a los vendedores, quienes ofrecen una infinidad de productos, entre los que alcanzan a distinguir vasijas, frutas, verduras, etcétera.
Llama la atención que el artista no establezca una diferenciación que indique su inclinación por uno de los dos frentes, lo que al mismo tiempo puede entenderse como el resultado de un enfrentamiento de conciudadanos, a pesar de la intervención de grupos extranjeros. ${ }^{24}$ En cuanto al conjunto de la imagen, hay una relación con La piedad en el desierto que había desarrollado Manuel Rodríguez Lozano en 1942, en la cárcel de Lecumberri, en la ciudad de México. En ésta se ve a una mujer sentada que sostiene con sus manos el cuerpo desfallecido de un hombre desnudo. La escena se sitúa en una zona desértica, lo que le imprime mayor dramatismo.

Dentro del proceso de reconstrucción del país que buscó reflejarse a través de las obras murales, pueden mencionarse las que Francisco Amighetti realizó en 1952 para la biblioteca del Policlínico de

$24 \mathrm{Al}$ respecto, véase Pérez, op. cit.; éste señala: "la insurrección estalló el 12 de marzo de 1948, cuando las fuerzas de Figueres, utilizando como base las montañas al sureste de San José, tomaron el aeropuerto de San Isidro del General. Con los aviones allí capturados se iniciaron los vuelos a Guatemala; así fueron llegando las armas y los combatientes de Centroamérica y el Caribe. Las fuerzas numerosas (las fuerzas regulares no superaban los 300 hombres) y mal armadas, intentaron un contraataque el 13 de marzo, pero los rebeldes resistieron y más bien consolidaron sus posiciones. Entre el 21 y el 23 de marzo, fuerzas irregulares del gobierno, apoyadas por trabajadores bananeros, atacaron San Isidro del General desde la costa, pero tampoco tuvieron éxito", p. 28. 


\section{Figura 3.}

\section{La medicina indígena}

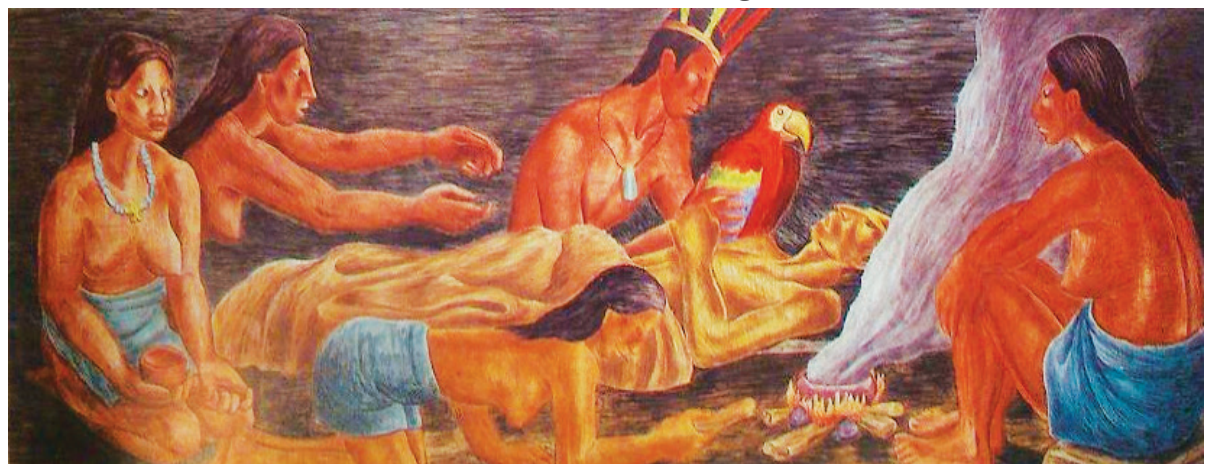

Autor: Francisco Amighetti, 1952. Imagen tomada de :http://www.vichitex.com/arte/historia_ cr/img_pintura/amighetti_medicina_indigena.jpg

la Caja Costarricense del Seguro Social, en las que contara nuevamente con la colaboración de Margarita Bertheau. ${ }^{25}$ El mural Medicina indígena alude a la medicina que practicaban los habitantes de Costa Rica antes de la colonización. Así, se ve a un enfermo tendido en el piso, y a su alrededor a cuatro mujeres y un hombre. Este último, que porta un penacho, pasa sobre el paciente una guacamaya, seguramente para absorber los males que aquejan al doliente. La mujer

25 Dentro de los datos biográficos que Montero, -op. cit.- incluye, menciona que las obras que Amighetti realizó en la Biblioteca del Policlínico correspondían a las siguientes temáticas: 1 . La medicina indígena de los primitivos habitantes de Costa Rica; 2. Uno de los aspectos sociales de la medicina en la actualidad, el médico rural trabajando con un fondo del típico campo criollo; 3. Retrato de Clodomiro Picado, sobre un fondo descriptivo de su obra científica; 4. Un retrato del doctor Durán y las instituciones de beneficencia, a cuya creación contribuyó con su amplia visión social. que está sentada del lado derecho, tiene al frente una vasija, que se encuentra en el fuego, de la que se desprende una intensa columna de humo.

El fuego de la fogata es avivado por la mujer que se halla casi tendida sobre el piso; con la mano derecha agrega un leño. Otra de las mujeres parece estar orando, al tiempo que dirige sus brazos hacia el enfermo. La última mujer está arrodillada y sostiene en su mano derecha una vasija y en la izquierda unas semillas, donde se ve la importancia que tenía la herbolaria para estas culturas. Sin embargo, por los colores oscuros que utiliza el artista, puede decirse que para éste simbolizaba una etapa de obscurantismo del que la humanidad había logrado salir con el transcurrir de los siglos, lo que no implicó que dichas prácticas fueran desterradas por completo. 


\section{Figura 4. \\ La medicina rural}

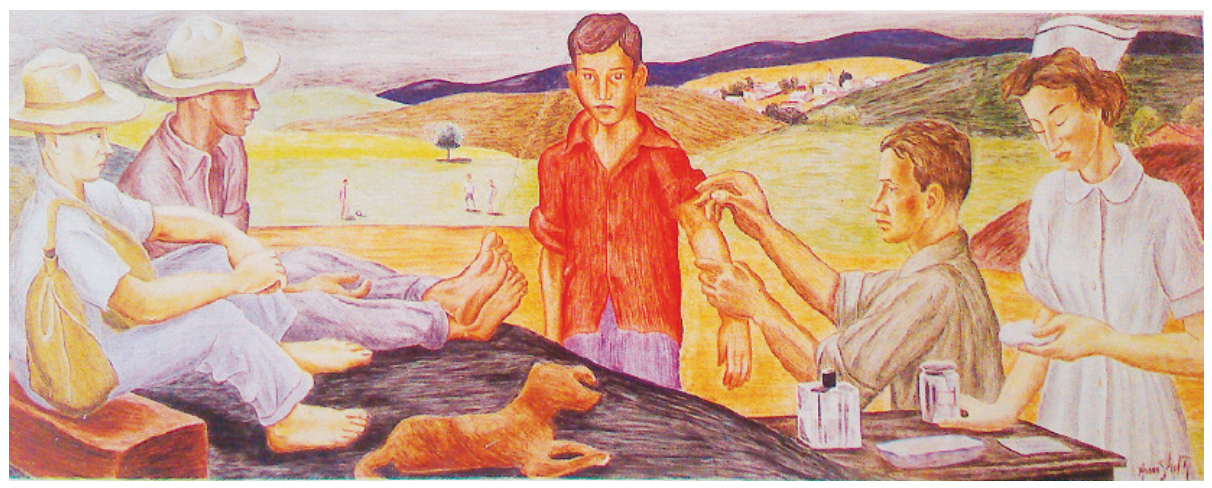

Autor: Francisco Amighetti, 1952. Imagen tomada de: http://www.franciscoamighetti.com/images/ obras/oleos/oleo2_b.jpg

En cuanto a la obra denominada La medicina rural, se aprecia en ella un completo cambio respecto de la obra Medicina indigena; pues ahora utiliza colores claros, para destacar los avances de la medicina. En primer plano, un médico rural está aplicando una vacuna a un joven de camisa roja. El galeno es asistido por una enfermera, quien parece llevar un control de las actividades que se realizan.

La escena es presenciada por dos hombres del campo, los cuales observan con atención las actividades que desarrolla el médico, como si trataran de despejar las dudas que éste les ocasiona. Como fondo de la imagen se representa el Eje Montañoso Central de Costa Rica, en donde sobresale un poblado, con lo que trata de enfatizarse lo lejano que es- taban algunas poblaciones de este tipo de servicios. Al mismo tiempo puede afirmarse que para el gobierno surgido de la Guerra Civil del 48 resultaba una prioridad tratar de llevar este tipo de servicios a las poblaciones más alejadas.

Dentro de las bondades que trataron de establecerse al terminar la Guerra Civil y la instauración de la Segunda República, pueden mencionarse la reorganización económica y social del país. Ello, como ya se vio, se hizo presente en las obras murales antes mencionadas; pero, sobre todo, se hará evidente en la realización del mural de La Segunda República realizado por Lucio Ranucci. ${ }^{26}$

26 Sobre este mural, digno de mención es el texto de Calderón, op. cit., pues no sólo realiza un estudio detallado de dicha obra, sino que es un testimonio que permitió su conservación. 
Resulta interesante que para el gobierno, en este momento precedido por José Figueres, el muralismo era un medio idóneo para difundir los logros de la recién concluida Guerra Civil. Cabe señalar que, con la construcción del aeropuerto, se propuso la elaboración de un mural en la sala de arribo de pasajeros. El mensaje que se buscaba que transmitiera la obra no era sólo hacia el interior, sino, sobre todo, hacia el exterior.

La convocatoria para elegir al artista que realizaría el mural se hizo a través del Ministerio de Obras Públicas, y se invitó a todos aquellos artistas que se encontraban en el país para presentar un proyecto:

La convocatoria estaba dirigida a los artistas nacionales y extranjeros residentes en Costa Rica. Como era de esperar, se presentaron muchos de los artistas reconocidos de la década de los cincuenta, entre ellos: Francisco Amighetti, Teodorico Quiros, Margarita Bertheau, Lola Fernández, el pintor italiano Lucio Ranucci [...] El trabajo escogido por el jurado fue el del artista Ranucci, quien finalmente realizó el mural en el aeropuerto tal como estipulaba el contrato con el gobierno. Inicialmente, el tema escogido por el artista fue una apología sobre la revolución del cuarenta y ocho. ${ }^{27}$

Llama la atención que el jurado se inclinó por el trabajo de este artista italiano - que había llegado a Costa Rica en 1951 para dirigir el Teatro Universitario-, ${ }^{28}$ sobre artistas como Francisco Amighetti o Margarita Bertheau, que ya habían practicado el muralismo. No obstante, puede decirse que si bien la intención era promover al país precisamente hacia el exterior, resultaba factible elegir a un artista que tendría que presentar una visión imparcial sobre este país.

Aunque, cabe recordar que con motivo de la toma presidencial de José Figueres Ferrer, en el Estadio Nacional en 1953, también se había utilizado un mural escenográfico, el cual fue obra precisamente de Lucio Ranucci, el tema de dicho mural fue "la guerra civil y los frutos que la contienda había logrado y defendía como un derecho de todos los costarricenses, por ejemplo la educación y la cultura, la salud, la agricultura, la alimentación, la construcción, la industria, los productos de exportación (el café), la familia y sobre todo [... el derecho y el respeto al voto universal". 29

27 Ibid., p. 31.

28 Sobre Lucio Ranucci, pueden verse: Lucio Ranucci, Lucio Ranucci. La mia odisea, Italia, L’Aquila, 2005; y Sylvie Murphy, Lucio Ranucci. Ou l aventure de l art, Francia, EDISUD, 1992.

29 Calderón, op. cit., p. 30. 


\section{Imagen 5. La Segunda República}

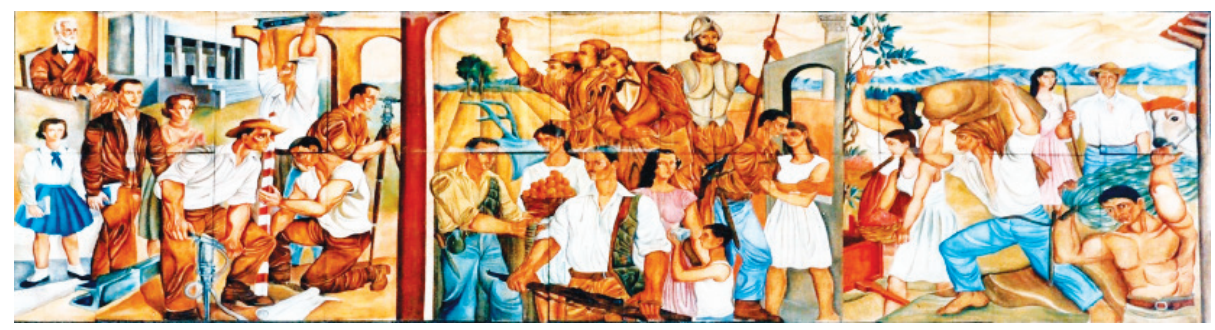

Autor: Lucio Ranucci, 1954. Imagen tomada de:https://i2.wp.com/elespiritudel48.org/wp-content/ uploads/2015/03/m2r.jpg

Por tanto, el mural de La Segunda República podría verse como la continuación de esa obra o, por lo menos, la reinterpretación de los temas que ya había abordado.

$\mathrm{Al}$ ser un tema histórico, llama la atención que un extranjero lograra plasmar lo que a su consideración eran los momentos más destacables de la historia de Costa Rica. ${ }^{30}$ No obstante, este aspecto lo superó al recibir apoyo de su mujer Olga Espinach, "quien le fue narrando la historia patria, para que él elaborando los dibujos de cada una de las partes del mural, le narra el periodo colonial,

30 Ibid., incluye el fragmento de una entrevista hecha a Olga Espinach, en la que recuerda que en un principio el artista tenía contemplado realizar una apología sobre la Guerra Civil, pero ella "le aconseja que es un tema muy delicado, por las consecuencias político-sociales que la guerra había generado entre los costarricenses y que además, por ser un acontecimiento que ha ocurrido tan solo seis años antes, podría despertar resentimientos y heridas muy profundas en especial en los perdedores de la Guerra Civil. Aconsejándole escoger el tema relacionado con la historia de Costa Rica”, p. 31. la independencia, la gesta heroica de 1956, la reforma educativa de don Mauro Fernández, la guerra civil de mil novecientos cuarenta y ocho, esta parte se enfatiza en la parte central del mural." ${ }^{{ }^{1}}$ La forma en que organizó dicha información recuerda en muchos sentidos la manera en que trabajaban los muralistas mexicanos, en especial Diego Rivera. ${ }^{32}$ Así, aunque aborda diversos temas en un solo mural, éste aparece divido en tres secciones. De izquierda a derecha podríamos decir que los temas generales son el progreso, la lucha y el comercio, aunque resulta claro que el punto de partida está en la sección central.

31 Ibid.

32 En diferentes obras, Diego Rivera recurrió a la unificación del pasado, presente y futuro. Aunque, sin duda, donde esto resulta más claro es en la Epopeya del pueblo mexicano, que plasmó en el Palacio Nacional de la ciudad de México. En éste incluso concentra los acontecimientos más importantes de la historia del país en la parte central del mural. 
Al centro del mural se encuentra la historia de Costa Rica, partiendo desde su etapa colonial, lo que queda ejemplificado con la figura del conquistador español, que porta su característica armadura. Delante de él se hallan Juan Santamaría, José María Cañas Escamilla y Juan Mora Porras, quienes se distinguirían en la Campaña Nacional de 1856-1857, por lo que eran reconocidos como héroes nacionales. Incluso, el primero de ellos porta una antorcha, como símbolo de libertad.

En primer plano se ven diferentes personajes que simbolizan la historia reciente; es decir, a los combatientes de la Guerra Civil, que el país acababa de enfrentar. "En esta escena se enfatizan los valores cívicos costarricenses, representados por dos grupos de personas, en primer lugar, un grupo de jóvenes que lucharon por los ideales de la Segunda República y representan a quienes defienden los derechos civiles; entre ellos, un niño que entrega una alcancía como símbolo de la solidaridad del costarricense que cooperó para la causa." ${ }^{33} \mathrm{El}$ apoyo de la sociedad hacia los combatientes se refuerza con la presencia de la mujer con una bandeja de frutos situada a la izquierda, pues simboliza que les ofrecían provisiones a pesar del abandono de los campos. El regreso a

33 Ibid., p. 35. la agricultura se daría con el retorno de los combatientes, lo que queda ejemplificado con la pareja que está a la derecha del mural.

Para la fecha en que se da la Guerra Civil, Costa Rica era un país mayoritariamente agrícola, por lo que será la principal actividad que trate de recuperarse después del conflicto armado, y será lo que se ve en el lado derecho del mural. Los dos productos que se destacan son el café y los plátanos. Ranucci establece, a través de la imagen, que en la actividad agrícola participaban tanto el hombre como la mujer, aunque la mujer parece estar encargada de las actividades ligeras, como es la recolección de los granos de café. Por su parte, los hombres aparecen cargando tanto los costales de granos, como los racimos de plátanos.

Además, un hombre y una mujer están contemplando las actividades agrícolas que se desarrollan; con ello, el artista señala a la familia como base del desarrollo agrícola, al mismo tiempo pueden verse como un símbolo de la fertilidad, pues, con esa unión se hace productiva la tierra. Como medio de transportación se emplea una carreta tirada por bueyes, aunque estos animales también eran utilizados para arar la tierra. De esta manera, varios de los elementos que están presentes en el mural de La agricultura 
de Francisco Amighetti se aprecían en esta obra. A través de esta sección, puede decirse que se ha puesto en marcha la reconstrucción del país, para lo que la actividad agrícola era lo principal.

En el lado izquierdo tenemos la visión del futuro o por lo menos, lo que resultaría de la reorganización del país. Así, "la educación la representa en primer lugar con la figura de Mauro Fernández, sentado en su Cátedra como líder de la reforma educativa de 1886 y tres estudiantes: una niña escolar y dos jóvenes universitarios, cubiertos por el Paraninfo de la Universidad, ubicada en el Barrio González Lahmann”. ${ }^{44}$ Por tanto, dentro del desarrollo que el artista consideraba que podía alcanzarse resultaba valido utilizar los elementos que hasta antes de la Guerra Civil podían considerarse benéficos y servirían para impulsar los logros del futuro.

Dichos adelantos quedan ejemplificados con los cuatro hombres que se encuentran taladrando, midiendo y sosteniendo vigas. De hecho, la propia construcción del aeropuerto, en el que se colocó este mural, era prueba de la importancia que tenían las obras públicas en la reconstrucción del país; pues, con ello, se buscaba introducir la modernidad en Costa Rica.

34 Ibid., p. 34.
A los murales hasta ahora mencionados se sumaron otros realizados por Francisco Amighetti; ${ }^{35}$ la propia Margarita Bertheau ejecutaría diferentes obras. ${ }^{36}$ A ellos se sumaron posteriormente Manuel de la Cruz González ${ }^{37}$ y César Valverde Vega. ${ }^{38}$ No obstante, a pesar de ello no logró consolidarse un movimiento fuerte como fue el caso de México, lo que ha llevado a Shifra M. Goldman a decir que: "en otras zonas, se realizaron relativamente pocos murales y no hubo oportunidades, como en México, para un movimiento mural nacional". ${ }^{39}$ En este sentido puede decirse que, si bien en un principio el gobierno patrocinó algunas obras, después de concluir la Guerra Civil de 1948, dicho patrocinio se interrumpió, lo cual no

35 En 1954 pintó dos murales para la Escuela Lincoln en San José.

36 Para el Colegio Saint Francis realizó la obra San Francisco con los pájaros; Aparición de San Francisco; Un ángel entrega a San Francisco un mensaje; San Francisco recibiendo los estigmas; y San Francisco entrega un mensaje a los monjes. Para la tienda La Dama elegante pintó Mujer que emerge de las aguas: Motivo de ballet; y Reinas de la noche. Para el Hospital de la Mujer Dr. Adolfo Carit Eva pintó Madre soltera.

37 Realizó en 1962, en las arcadas en San José la obra Mural Espacial, que se caracteriza por ser una obra abstracta, con lo que se marca un nuevo rumbo en la realización de murales.

38 Sobre la obra de este artista, se ha publicado: César Valverde Vega, Los murales de César Valverde, San José de Costa Rica, L'Atelier, 1990.

39 Shifra M. Goldman, Perspectivas artísticas del Continente Americano. Arte y cambio social en América Latina y Estados Unidos en el siglo xx, México, Universidad Autónoma de la Ciudad de México-Instituto Nacional de Bellas Artes, 2008, p. 173. 
significa que la realización de murales

hubiera desaparecido, hasta la fecha se siguen creando, y sería necesario hacer un estudio más profundo y extenso para seguir los diversos caminos que siguieron, después bajo la influencia del muralismo mexicano. 